\title{
TRITERPENOS E ALCALÓIDE TIPO CANTINONA DOS GALHOS DE Simaba polyphylla (CAVALCANTE) W.W. THOMAS (SIMAROUBACEAE)
}

\author{
Rita de Cássia Guedes Saraiva* e Angelo C. Pinto \\ Instituto de Química, Universidade Federal do Rio de Janeiro, Centro de Tecnologia, Bloco A, Cidade Universitária, \\ 21945-970 Rio de Janeiro - RJ

\section{Sergio Massayoshi Nunomura e Adrian Martin Pohlit} \\ Coordenação de Pesquisas em Produtos Naturais, Instituto Nacional de Pesquisas da Amazônia, Av. André Araújo, 2936, \\ 69083-000 Manaus - AM
}

Recebido em 28/3/05; aceito em 2/8/05; publicado na web em 13/1/06

\begin{abstract}
TRITERPENES AND A CANTHINONE ALKALOID FROM THE STEMS OF Simaba polyphylla (CAVALCANTE) W.W. THOMAS (SIMAROUBACEAE). Simaba polyphylla is a small tree found in the Amazon region, known by the common names "marupazinho" or "serve para tudo". It is used in traditional medicine for the treatment of fevers. This work describes the phytochemical study of the hexane extract and chloroform fraction obtained by partitioning the methanol extract of stems, which led to isolation and identification of the triterpenes niloticin, dyhidroniloticin, taraxerone and of the cytotoxic alkaloid 9-methoxy-canthin-6-one. These compounds are described for the first time in S. polyphylla.
\end{abstract}

Keywords: tirucallane; taraxerone; 9-methoxy-canthin-6-one.

\section{INTRODUÇÃO}

A espécie Simaba polyphylla, da família Simaroubaceae, caracteriza-se como um arbusto de 3-4 $\mathrm{m}$ ou uma arvoreta de 5-10 $\mathrm{m}$ de altura e é nativa da região amazônica, onde ocorre normalmente em mata de terra-firme ${ }^{1}$. As espécies da família Simaroubaceae são conhecidas por suas propriedades medicinais utilizadas tradicionalmente para tratamento da malária, e também como anti-helmínticos, antitumorais, entre outros ${ }^{2}$. Os constituintes químicos comuns encontrados em espécies do gênero Simaba são quassinóides ${ }^{3-5}$ e alcalóides do tipo cantinona ${ }^{6,7}$. A espécie S. polyphylla é conhecida popularmente na Amazônia como "serve para tudo" ou "marupazinho" e é usada para tratamento de diversos tipos de febre ${ }^{1}$ Além disso, o extrato metanólico dos galhos dessa espécie revelou-se muito ativo contra larvas de Aedes aegypti $i^{8}$. Estudos anteriores dessa espécie resultaram no isolamento de um único alcalóide do tipo cantinona identificado como cantin-2,6-diona ${ }^{9}(\mathbf{1})$. O presente trabalho descreve o isolamento de um outro alcalóide, a 9-metoxi-cantin-6-ona (2), bem como os triterpenos niloticina (3), diidroniloticina (4) e taraxerona (5).

\section{PARTE EXPERIMENTAL}

Para obtenção de pontos de fusão foi utilizado aparelho Mel-Temp II (Laboratory Devices Inc., EUA), com amostras acondicionadas em capilares de vidro. Os valores não foram corrigidos.

Os espectros de infravermelho foram obtidos em aparelho Nicolet Magna IR 760, com pastilhas comprimidas em KBr anidro. Os valores para as absorções foram referidos em números de ondas $\left(\mathrm{cm}^{-1}\right)$ e os espectros foram calibrados com filmes de poliestireno de $0,05 \mathrm{~mm}$ de espessura nas freqüências de absorção: 3030; 2925; $1601 ; 1493 ; 757$ e $698 \mathrm{~cm}^{-1}$.

*e-mail: ritasaraiva@uol.com.br
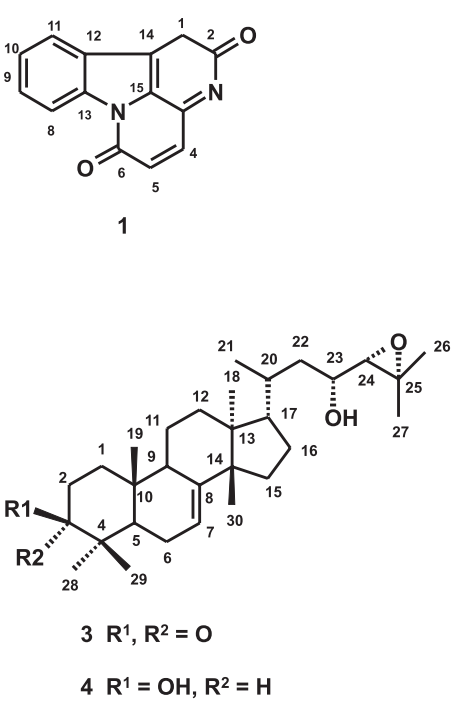

Figura 1. Substâncias isoladas de Simaba polyphylla

Os espectros de RMN foram obtidos em aparelho Bruker, modelo DRX-200, operando a $200 \mathrm{MHz}$ para $\mathrm{RMN}{ }^{1} \mathrm{H}$ e a $50 \mathrm{MHz}$ para $\mathrm{RMN}{ }^{13} \mathrm{C}$.

Para obtenção dos espectros de massas foi usado cromatógrafo a gás de alta resolução Hewlett-Packard 6890 Series GC System acoplado ao espectrômetro de massas do tipo HewlettPackard HP 5973 Mass Selective Detector, controlados por computador através do software Standard ChemStation G1701AA versão A03 (1996). Foi utilizada a coluna DB5, com 25 m x 0,2 $\mathrm{mm} \times 0,11 \mu \mathrm{m}$ de espessura de filme. Temperatura do injetor: $270{ }^{\circ} \mathrm{C}$ com divisor de fluxo (1/20); gás de arraste: He. Temperatura inicial do forno $80{ }^{\circ} \mathrm{C}$, rampa de aquecimento a $8{ }^{\circ} \mathrm{C} / \mathrm{min}$ até $290{ }^{\circ} \mathrm{C}$, permanecendo nessa temperatura por $5 \mathrm{~min}$. O espectro de massas foi obtido por impacto de elétrons a $70 \mathrm{eV}$, cujos fragmentos foram descritos como relação entre unidade 
de massa atômica (uma) e a carga dos mesmos $(\mathrm{m} / \mathrm{z})$. A abundância relativa de cada fragmento foi expressa em percentagem relativa ao pico base.

\section{Material vegetal}

A espécie Simaba polyphylla foi coletada na Reserva Ducke do INPA no Amazonas, em fevereiro de 2002. Foram coletadas as partes aéreas da planta, separadas em folhas e galhos. 2,0 kg dos galhos foram secados e posteriormente moídos para extração. As exsicatas dos espécimes coletados encontram-se no herbário do INPA identificadas com os números 3003 e 3209.

\section{Extração e fracionamento}

Os galhos secos e moídos $(2,0 \mathrm{~kg}$ ) foram macerados durante sete dias em hexano à temperatura ambiente. $\mathrm{O}$ processo foi repetido por mais duas vezes e os extratos concentrados foram reunidos, totalizando $22,1 \mathrm{~g}$ de extrato. Após a extração em hexano, o material vegetal foi submetido a três macerações sucessivas com metanol, à temperatura ambiente, durante sete dias que resultou em 71,2 g de extrato. Parte desse extrato $(66,68 \mathrm{~g})$ foi particionado com $\mathrm{CHCl}_{3}$, que após concentração rendeu 33,7 $\mathrm{g}$ e posteriormente com AcOEt, rendendo em 11,8 g. O extrato hexânico $(17,9 \mathrm{~g})$ foi fracionado em coluna de gel de sílica 60 , utilizando como eluente gradiente de hexano e acetato de etila (AcOEt). A separação cromatográfica forneceu, após análise em CCD, 18 frações, das quais as frações 9 e 10, eluídas em hexano/ AcOEt (8:2) e (7:3), foram recristalizadas em AcOEt, resultando no isolamento de 201,7 e $177,8 \mathrm{mg}$, dos triterpenos niloticina (3) e diidroniloticina (4), respectivamente. A fração clorofórmica $(28,5 \mathrm{~g})$ foi separada em coluna filtrante utilizando como eluente hexano $(100 \%)$ seguido de $\mathrm{CHCl}_{3}(100 \%)$, gradiente $\mathrm{CHCl}_{3} /$ $\mathrm{MeOH}$ e $\mathrm{MeOH}(100 \%)$. Dessa separação resultaram 15 frações. As frações 5 e 6 , eluídas em $100 \%$ de $\mathrm{CHCl}_{3}$ foram recristalizadas em metanol, resultando no isolamento de $18 \mathrm{mg}$ do triterpeno taraxerona $(\mathbf{5})$.

A fração 8, eluída em $\mathrm{CHCl}_{3} / \mathrm{MeOH}$ (99:1), foi submetida a um novo fracionamento cromatográfico utilizando como eluente o sistema gradiente $\mathrm{CHCl}_{3} / \mathrm{MeOH}$, rendendo 41 frações. As frações de 17 a 19, eluídas em $\mathrm{CHCl}_{3} / \mathrm{MeOH}$ (98:2), foram reunidas e submetidas à separação cromatográfica em coluna de sílica flash usando sistema de eluição gradiente hexano/ $/ \mathrm{CHCl}_{3} / \mathrm{MeOH}$. Dessa separação, as frações de 30 a 33, eluídas em $\mathrm{Hex} / \mathrm{CHCl}_{3} / \mathrm{MeOH}$ (55:44:1), foram reunidas e recristalizadas em $\mathrm{MeOH}$ a quente que resultou em 11,0 mg do alcalóide 9-metoxi-cantin-6-ona (2).

\section{Caracterização da 9-metoxi-cantin-6-ona (2)}

Cristais amarelos, PF. $175-176{ }^{\circ} \mathrm{C}$ (lit. $\left.{ }^{7} 175-176{ }^{\circ} \mathrm{C}\right), \mathrm{IV}(\mathrm{KBr})$ $\mathrm{v}_{\max } \mathrm{cm}^{-1}: 1667,1633,1608,1493,1275,1223,1152,1030,844$, 815, 619, 547. EM-IE $(\mathrm{m} / \mathrm{z}): 250\left[\mathrm{M}^{+}\right](100), 235(1,3), 221(21,3)$, 207 (32,0), 192 (4,0), 179 (17,3), $153(9,3), 126(6,6), 125(8,0)$. $\mathrm{RMN}^{1} \mathrm{H}$ e ${ }^{13} \mathrm{C}$ : Tabela 3.

\section{Caracterização da niloticina (3)}

Cristais incolores, PF $145-147{ }^{\circ} \mathrm{C}$ (lit. $\left.{ }^{10} 147{ }^{\circ} \mathrm{C}\right)$, IV (KBr) $v_{\max } \mathrm{cm}^{-1}: 3468,2969,1705,1664,1460,1381,1366,1291,1061$, 972, 908, 813. EM-IE (m/z): $384\left[\mathrm{M}^{+}-\mathrm{C}_{4} \mathrm{H}_{8} \mathrm{O}\right](18), 369$ (100), 351 (38), 325 (54), 271 (17), 213 (10), 147 (22), 133 (26) e 55 (51). RMN ${ }^{1} \mathrm{H}$ e ${ }^{13} \mathrm{C}$ : Tabela 1.

\section{Caracterização da diidroniloticina (4)}

Cristais incolores, PF $173-174{ }^{\circ} \mathrm{C}$ (lit. $\left.{ }^{10} 174{ }^{\circ} \mathrm{C}\right)$, IV (KBr) $\mathrm{v}_{\max } \mathrm{cm}^{-1}: 3458,2951,2880,1651,1462,1443,1380,1035,990$. EM-IE (m/z): $429\left[\mathrm{M}^{+}-\mathrm{C}_{2} \mathrm{H}_{5}\right]$ (4), 386 (26), 371 (100), 353 (83), 335 (22), 309 (18), 281 (26), 253 (15), 207 (74), 147 (46), 135 (47), 121 (63), 105 (68), 55 (77). RMN ${ }^{1} \mathrm{H}$ e ${ }^{13} \mathrm{C}$ : Tabela 2.

\section{Caracterização da taraxerona (5)}

Cristais incolores, PF 220-221 ${ }^{\circ} \mathrm{C}$ (lit. ${ }^{11}$ 218-221 ${ }^{\circ} \mathrm{C}$ ), IV (KBr) $\mathrm{v}_{\max } \mathrm{cm}^{-1}: 3048,2938,1709,1640,1376,995$ e 816 . EM-IE $(\mathrm{m} / \mathrm{z})$ : $424\left[\mathrm{M}^{+}\right]$(33), 409 (22), 300 (99), 285 (74), 272 (15), 257 (12), 204 (100), 189 (34), 133 (65). RMN ${ }^{1} \mathrm{H}\left(200 \mathrm{MHz}, \mathrm{CDCl}_{3}\right): \delta 1,90$ (m, H-1); 2,58 (ddd; $J=7,1 ; 12,0$ e $15,8 \mathrm{~Hz}, \mathrm{H}-2$ ); 2,33 (ddd; $J=$ $3,4 ; 6,1$ e $15,8 \mathrm{~Hz}, \mathrm{H}-2) ; 1,31$ (m, H-5); 5,56 (dd; $J=3,0$ e $8,1 \mathrm{~Hz}$, H-15); 1,08 (s, Me-23); 1,07 (s, Me-24); 1,08 (s, Me-25); 0,83 (s, Me-26); 1,14 (s, Me-27); 0,91 (s, Me-28); 0,95 (s, Me-29); 0,91 (s, $\mathrm{Me}-30)$; RMN ${ }^{13} \mathrm{C}\left(50 \mathrm{MHz}, \mathrm{CDCl}_{3}\right)$ : $\delta$ 38,5 (C-1); 34,2 (C-2); 217,7 (C-3); 48,8 (C-4); 55,9 (C-5); 20,1 (C-6); 35,2 (C-7); 37,9 (C-8); 48,9 (C-9); 35,8 (C-10); 17,5 (C-11); 36,8 (C-12); 37,7 (C13); 157,3 (C-14); 117,3 (C-15); 37,8 (C-16); 37,7 (C-17); 48,9 (C-18); 40,8 (C-19); 28,9 (C-20); 33,7 (C-21); 33,2 (C-22); 26,2 (C-23); 21,6 (C-24); 14,9 (C-25); 30,0 (C-26); 25,7 (C-27); 30,1 (C-28); 33,5 (C-29); 21,5 (C-30).

\section{RESULTADOS E DISCUSSÃO}

O espectro de IV do triterpeno niloticina (3) apresentou uma banda em 3468 e $1705 \mathrm{~cm}^{-1}$, consistente com a presença de grupos hidroxila e carbonila, respectivamente. $\mathrm{O}$ espectro de massas não exibiu o íon molecular $\mathrm{M}^{+}(456 \mathrm{~m} / z)$, porém as fragmentações são compatíveis com o espectro de massas da niloticina $(\mathrm{m} / \mathrm{z}, 384,369$, 351 indicando a perda sucessiva dos grupamentos 2,2-dimetiloxirano, metil e água da cadeia lateral do triterpeno). A estrutura de 3 foi confirmada por análise de $\mathrm{RMN}{ }^{1} \mathrm{H}$ e ${ }^{13} \mathrm{C}$. A Tabela 1 descreve os deslocamentos químicos de $\mathrm{RMN}{ }^{1} \mathrm{H}$ e $\mathrm{RMN}{ }^{13} \mathrm{C}$ atribuídos após análise dos espectros mono e bidimensionais HOMOCOSY, HMBC e HMQC, além de comparação com dados publicados anteriormente $\mathrm{e}^{10,12}$.

Comparando-se com os dados da literatura ${ }^{10}$, foram realizadas algumas reatribuições de deslocamentos químicos. Os sinais em $\delta$ 21,7 e 12,9 foram atribuídos aos carbonos metílicos 18 e 19, respectivamente. Esses puderam ser confirmados através das correlações C-H à longa distância observada para a Me-18 com os carbonos C-13 e C-14. Também foi observada a correlação à longa distância do carbono C-5 com a metila C-19.

$\mathrm{O}$ triterpeno diidroniloticina (4) apresentou no espectro de IV uma banda intensa e larga em $3458 \mathrm{~cm}^{-1}$ referente aos grupos hidroxílicos na molécula. Uma banda fraca em $1651 \mathrm{~cm}^{-1}$ foi atribuída à presença de ligação dupla entre carbonos. Do mesmo modo que a substância $\mathbf{3}$, a substância $\mathbf{4}$ não apresentou o íon molecular $\mathrm{M}^{+}(458 \mathrm{~m} / \mathrm{z})$, no espectro de massas. Entretanto, as fragmentações observadas são compatíveis com os dados da literatura ${ }^{10}$. A estrutura foi confirmada por análise dos deslocamentos químicos de $\mathrm{RMN}{ }^{1} \mathrm{H}$ e ${ }^{13} \mathrm{C}$ (Tabela 2) e das correlações observadas nos espectros bidimensionais de HOMOCOSY, HMQC e HMBC. Alguns deslocamentos químicos de carbono tais como o C-5 $(\delta 53,4)$ e C-17 $(\delta 50,8)$ apresentam uma significativa diferença dos dados apresentados pela literatura. $\mathrm{O}$ deslocamento químico de C-5 foi confirmado através das correlações à longa distância observadas em HMBC do C-5 com a metila C-28 e C-19. O carbono C-17 apresenta correlação no HMQC com o hidrogênio em $\delta 1,38$. Este apresenta correlação no HMBC $\left({ }^{3} \mathrm{~J}_{\mathrm{CH}}\right)$ com a metila C-21. As metilas C-18 e C-19 também foram reatribuídas, com base nas correlações à longa distância observadas. Os hidrogênios da metila Me-19 apresen- 
Tabela 1. Deslocamentos químicos de $\mathrm{RMN}{ }^{1} \mathrm{H}$ e ${ }^{13} \mathrm{C}\left(\mathrm{CDCl}_{3}\right)$ observados para a substância 3 e dados da literatura para a niloticina

\begin{tabular}{|c|c|c|c|c|c|}
\hline $\mathrm{C} / \mathrm{H}$ & $\delta \mathrm{C}(50 \mathrm{MHz})$ & $\delta \mathrm{H}(200 \mathrm{MHz})$ & $\operatorname{HMBC}\left(2,3 J_{\mathrm{CH}}\right)$ & Lit. $^{10}(\delta \mathrm{C})^{\mathrm{a}}$ & Lit. $^{10}(\delta \mathrm{H})^{\mathrm{a}}$ \\
\hline \multirow[t]{2}{*}{1} & 38,6 & $1,40(\mathrm{~m})$ & 2,76 & 38,1 & $1,40(\mathrm{~m})$ \\
\hline & & $1,96(\mathrm{~m})$ & & & $2,02(\mathrm{~m})$ \\
\hline \multirow[t]{2}{*}{2} & 35,0 & $2,24(\mathrm{t} ; 3,5 ; 3,5 ; 3,5)$ & 1,96 & 34,4 & $2,27(\mathrm{~m})$ \\
\hline & & $2,76(\mathrm{dt} ; 14,2 ; 5,2)$ & & & $2,75(\mathrm{td} ; 14,5 ; 5,5)$ \\
\hline 3 & 217,0 & - & 2,$76 ; 1,12$ & 215,1 & ND \\
\hline 4 & 48,0 & - & 1,$05 ; 2,08$ & 47,4 & ND \\
\hline 5 & 52,4 & $1,72(\mathrm{~m})$ & 1,$12 ; 5,31 ; 1,05$ & 52,0 & ND \\
\hline \multirow[t]{2}{*}{6} & 24,6 & $2,08(\mathrm{~m})$ & - & 24,0 & $2,27(\mathrm{~m})$ \\
\hline & & & & & $2,08(\mathrm{~m})$ \\
\hline 7 & 118,1 & $5,31(\mathrm{~d} ; 3,0)$ & - & 117,6 & $5,27(\mathrm{td} ; 3,5 ; 3,0)$ \\
\hline 8 & 145,9 & - & - & 145,3 & ND \\
\hline 9 & 48,6 & $2,28(\mathrm{~m})$ & 1,$72 ; 1,65 ; 1,89$ & 48,3 & $2,02(\mathrm{~m})$ \\
\hline 10 & 35,1 & - & 2,24 & 34,6 & ND \\
\hline 11 & 19,1 & $1,60(\mathrm{~m})$ & - & 17,9 & ND \\
\hline \multirow[t]{2}{*}{12} & 33,8 & $1,65(\mathrm{~m})$ & - & 33,3 & ND \\
\hline & & $1,89(\mathrm{~m})$ & & & \\
\hline 13 & 43,7 & - & 0,82 & 43,2 & ND \\
\hline 14 & 51,3 & - & 0,82 & 50,8 & ND \\
\hline 15 & 34,1 & $1,46(\mathrm{~m})$ & - & 33,7 & ND \\
\hline \multirow[t]{2}{*}{16} & 29,0 & $1,64(\mathrm{~m})$ & - & 28,3 & ND \\
\hline & & $2,00(\mathrm{~m})$ & & & \\
\hline 17 & 53,4 & $1,57(\mathrm{~m})$ & - & 52,9 & ND \\
\hline 18 & 21,7 & $0,82(\mathrm{~s})$ & - & 12,4 & $0,94(s)$ \\
\hline 19 & 12,9 & $1,00(\mathrm{~s})$ & 1,72 & 19,4 & $0,75(\mathrm{~s})$ \\
\hline 20 & 33,6 & $1,86(\mathrm{~m})$ & - & 33,2 & $1,20(\mathrm{~m})$ \\
\hline 21 & 20,0 & $0,95(\mathrm{~s})$ & - & 19,6 & $0,89(\mathrm{~s})$ \\
\hline \multirow[t]{2}{*}{22} & 41,0 & $1,43(\mathrm{~m})$ & - & 40,4 & $1,40(\mathrm{~m}) 1,55(\mathrm{~m})$ \\
\hline & & $1,67(\mathrm{~m})$ & & & \\
\hline 23 & 69,3 & $3,58(\mathrm{ddd} ; 5,0 ; 8,5 ; 8,0)$ & - & 68,8 & $3,49(\mathrm{dtd} ; 8,4 ; 5,2 ; 2,7)$ \\
\hline 24 & 68,6 & $2,66(\mathrm{~d} ; 8,0)$ & - & 68,2 & $2,64(\mathrm{~d} ; 8,4)$ \\
\hline 25 & 60,3 & - & - & 59,4 & ND \\
\hline 26 & 19,9 & $1,33(\mathrm{~s})$ & - & 21,2 & $1,25(\mathrm{~s})$ \\
\hline 27 & 24,6 & $1,32(\mathrm{~s})$ & - & 24,2 & $1,26(\mathrm{~s})$ \\
\hline 28 & 25,0 & $1,05(\mathrm{~s})$ & 1,$12 ; 1,72$ & 24,5 & $1,05(\mathrm{~s})$ \\
\hline 29 & 21,9 & $1,12(\mathrm{~s})$ & 1,$05 ; 1,72$ & 21,4 & $0,95(\mathrm{~s})$ \\
\hline 30 & 27,5 & $1,02(\mathrm{~s})$ & 1,$46 ; 2,00$ & 27,0 & $0,95(\mathrm{~s})$ \\
\hline
\end{tabular}

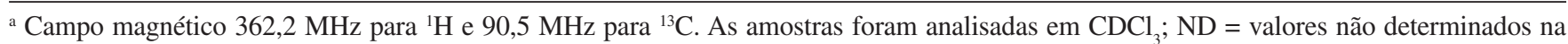
literatura ${ }^{10}$.

tam correlação à longa distância com o C-10 $\left({ }^{2} \mathrm{~J}_{\mathrm{CH}}\right), \mathrm{C}-9\left({ }^{3} \mathrm{~J}_{\mathrm{CH}}\right)$, C-1 $\left({ }^{3} \mathrm{~J}_{\mathrm{CH}}\right)$ e C-5 $\left({ }^{3} \mathrm{~J}_{\mathrm{CH}}\right)$ e os hidrogênios da metila C-18 apresentam correlação no HMBC com os carbonos C-12 $\left({ }^{3} \mathrm{~J}_{\mathrm{CH}}\right)$ e C-13 $\left({ }^{2} \mathrm{~J}_{\mathrm{CH}}\right)$.

Os dados de $\mathrm{RMN}{ }^{13} \mathrm{C}$ e ${ }^{1} \mathrm{H}$ do triterpeno taraxerona (5), isolado da fração clorofórmica dos galhos de $S$. polyphylla, foram atribuídos inicialmente por comparação com dados publicados ${ }^{11-13}$. A atribuição dos deslocamentos químicos de (5) foi confirmada através das correlações observadas em espectros de HOMOCOSY, HMQC e HMBC. No espectro de IV, as bandas em 3048 e 1640 $\mathrm{cm}^{-1}$ são referentes à deformação axial e angular da ligação dupla em C-14. A frequiência de absorção em $1709 \mathrm{~cm}^{-1}$ corresponde à deformação axial da carbonila presente na posição C-3.

O alcalóide 9-metoxi-cantinona (2) apresentou-se como cristais amarelos com PF e espectro de massas compatíveis com dados descritos na literatura ${ }^{7}$. Através da análise do espectro de RMN de ${ }^{1} \mathrm{H}$ sugere-se que a metoxila esteja ligada à posição 9 , através das constantes de acoplamento entre os hidrogênios H-8 e H-10 (2,4 $\mathrm{Hz}$ ), com acoplamento entre hidrogênios com relação meta. Além disso, o hidrogênio H-10 apresenta constante de acoplamento adici- onal de $8,5 \mathrm{~Hz}$ corroborada pela constante de $8,5 \mathrm{~Hz}$ observada para o hidrogênio H-11. A estrutura foi confirmada através da análise dos espectros bidimensionais HOMOCOSY, HMQC e HMBC. As correlações à longa distância entre C-9 e os hidrogênios da metoxila e o hidrogênio $\mathrm{H}-10 \mathrm{com}$ os carbonos $\mathrm{C}-12$ e C-8, no espectro de HMBC, confirmam a estrutura como 9-metoxi-cantin6-ona. Os deslocamentos químicos de $\mathrm{RMN}$ de ${ }^{1} \mathrm{H}$ e $\mathrm{C}^{13}$ são descritos na Tabela 3.

O alcalóide 9-metoxi-cantin-6-ona foi isolado anteriormente de Eurycoma longifolia e apresentou forte atividade citotóxica em ensaios in vitro com células tumorais tipo MCF-7 (câncer de mama) e A-549 (câncer de pulmão) ${ }^{14}$.

\section{CONCLUSÃO}

O isolamento de triterpenos tetracíclicos do tipo tirucalano com insaturação na posição C-7, como registrado nesse trabalho, é comum em espécies da família Simaroubaceae ${ }^{15,16}$, visto que triterpenos dessa classe são precursores de quassinóides ${ }^{17}$, os principais marcadores quimiotaxonômicos dessa família. A presença 
Tabela 2. Deslocamentos químicos observados de $\mathrm{RMN}{ }^{1} \mathrm{H}$ e ${ }^{13} \mathrm{C}$ para a substância 4 em $\mathrm{CDCl}_{3}$

\begin{tabular}{|c|c|c|c|c|c|}
\hline $\mathrm{C} / \mathrm{H}$ & $\delta \mathrm{C}(50 \mathrm{MHz})$ & $\delta \mathrm{H}(200 \mathrm{MHz})$ & $\operatorname{HMBC}\left({ }^{2,3} \mathrm{~J}_{\mathrm{CH}}\right)$ & Lit. $^{10}(\delta \mathrm{C})^{\mathrm{b}}$ & Lit. $^{10}(\delta \mathrm{H})^{\mathrm{b}}$ \\
\hline \multirow[t]{2}{*}{1} & 37,3 & $1,73(\mathrm{~m})$ & 0,74 & 37,3 & ND \\
\hline & & $1,66(\mathrm{~m})$ & & & \\
\hline \multirow[t]{2}{*}{2} & 27,8 & $1,55(\mathrm{~m})$ & - & 28,8 & ND \\
\hline & & $1,64(\mathrm{~m})$ & & & \\
\hline 3 & 79,3 & $3,24(\mathrm{dd} ; 4,4 ; 11,0)$ & 0,86 & 79,3 & $3,25(\mathrm{dd} ; 10,0 ; 5,0)$ \\
\hline 4 & 39,0 & - & 0,86 & 39,0 & ND \\
\hline 5 & 53,4 & $1,56(\mathrm{~m})$ & 0,$81 ; 0,74$ & 59,7 & ND \\
\hline \multirow[t]{2}{*}{6} & 24,0 & $1,96(\mathrm{~m})$ & 5,26 & 24,0 & $2,00(\mathrm{~m})$ \\
\hline & & $2,19(\mathrm{~m})$ & & & $2,30(\mathrm{~m})$ \\
\hline 7 & 118,2 & $5,26(\mathrm{dd} ; 3,0 ; 6,6)$ & 1,$45 ; 2,19$ & 118,1 & $5,27(\mathrm{td} ; 3,2 ; 3,0)$ \\
\hline 8 & 145,7 & - & 2,$19 ; 0,98$ & 145,6 & ND \\
\hline \multirow[t]{2}{*}{9} & 49,1 & $2,19(\mathrm{~m})$ & 5,$26 ; 0,74$ & 49,1 & $2,00(\mathrm{~m})$ \\
\hline & & & & & $2,30(\mathrm{~m})$ \\
\hline 10 & 35,0 & - & 2,$19 ; 1,66 ; 1,73 ; 0,74$ & 35,0 & ND \\
\hline \multirow[t]{2}{*}{11} & 18,2 & $1,51(\mathrm{~m})$ & - & 18,2 & ND \\
\hline & & $1,56(\mathrm{~m})$ & & & \\
\hline 12 & 33,8 & $1,53(\mathrm{~m})$ & 0,94 & 34,1 & ND \\
\hline 13 & 43,7 & - & 2,$10 ; 0,94$ & 43,7 & ND \\
\hline 14 & 51,3 & - & 0,$98 ; 5,26$ & 51,2 & ND \\
\hline 15 & 34,1 & $1,45(\mathrm{~m})$ & 2,10 & 34,1 & ND \\
\hline \multirow[t]{2}{*}{16} & 28,9 & $2,04(\mathrm{~m})$ & - & 27,7 & ND \\
\hline & & $2,10(\mathrm{~m})$ & & & \\
\hline 17 & 50,8 & $1,38(\mathrm{~m})$ & - & 53,3 & ND \\
\hline 18 & 19,9 & $0,94(s)$ & - & 13,1 & $0,81(\mathrm{~s})$ \\
\hline 19 & 13,2 & $0,74(\mathrm{~s})$ & - & 19,8 & 0,75 \\
\hline 20 & 33,7 & $1,76(\mathrm{~m})$ & - & 33,7 & ND \\
\hline 21 & 20,1 & $0,97(s)$ & 1,38 & 20,0 & ND \\
\hline 22 & 40,9 & $1,40(\mathrm{~m})$ & - & 40,9 & ND \\
\hline 23 & 69,4 & $3,57(\mathrm{ddd} ; 5,2 ; 8,1 ; 8,3)$ & 2,66 & 69,4 & ND \\
\hline 24 & 68,7 & $2,66(\mathrm{~d} ; 8,3)$ & 3,57 & 68,5 & $2,66(\mathrm{~d} ; 8,2)$ \\
\hline 25 & 60,4 & - & - & 60,2 & ND \\
\hline 26 & 25,0 & $1,33(\mathrm{~s})$ & - & 21,7 & $1,34(\mathrm{~s})$ \\
\hline 27 & 27,8 & $1,32(\mathrm{~s})$ & - & 24,9 & $1,32(\mathrm{~s})$ \\
\hline 28 & 21,9 & 0,81 (s) & - & 27,3 & 0,99 (s) \\
\hline 29 & 14,9 & 0,86 (s) & - & 14,8 & $0,86(\mathrm{~s})$ \\
\hline 30 & 27,3 & 0,98 (s) & - & 27,9 & 0,97 (s) \\
\hline
\end{tabular}

${ }^{\mathrm{b}}$ Campo magnético de $250 \mathrm{MHz}$ para ${ }^{1} \mathrm{H}$ e $62,5 \mathrm{MHz}$ para ${ }^{13} \mathrm{C}$. Amostras analisadas em $\mathrm{CDCl}_{3}$; $\mathrm{ND}=$ valores não determinados na literatura ${ }^{10}$.

Tabela 3. Deslocamentos químicos de $\mathrm{RMN}{ }^{1} \mathrm{H}$ e ${ }^{13} \mathrm{C}$ observados para a 9-metoxi-cantin-6-ona (2)

\begin{tabular}{|c|c|c|c|c|}
\hline $\mathrm{C} / \mathrm{H}$ & $\delta \mathrm{C}(50 \mathrm{MHz})$ & $\delta \mathrm{H}(200 \mathrm{MHz})$ & $\operatorname{HMBC}\left(2,3 \mathrm{~J}_{\mathrm{CH}}\right)$ & $\delta \mathrm{H}(100 \mathrm{MHz}) /\left(\right.$ Lit. $\left.^{7}\right)$ \\
\hline 1 & 115,7 & $7,83(\mathrm{~d} ; 5,2)$ & 8,75 & $7,76(\mathrm{~d} ; 5,0)$ \\
\hline 2 & 146,1 & $8,75(\mathrm{~d} ; 5,2)$ & 7,83 & $8,72(\mathrm{~d} ; 5,0)$ \\
\hline 4 & 140,0 & $8,00(\mathrm{~d} ; 10,0)$ & - & 7,96 (d; 10,0) \\
\hline 5 & 128,7 & $6,94(\mathrm{~d} ; 10,0)$ & - & $6,90(\mathrm{~d} ; 10,0)$ \\
\hline 6 & 159,9 & - & 8,00 & - \\
\hline 8 & 101,5 & $8,18(\mathrm{~d} ; 2,4)$ & 7,06 & $8,13(\mathrm{~d} ; 2,0)$ \\
\hline 9 & 162,7 & - & 8,$18 ; 3,98 ; 7,93$ & - \\
\hline 10 & 114,3 & $7,06(\mathrm{dd} ; 2,4 ; 8,5)$ & - & $7,01(\mathrm{dd} ; 7,0 ; 2,0)$ \\
\hline 11 & 123,5 & $7,93(\mathrm{~d} ; 8,5)$ & - & $7,85(\mathrm{~d} ; 7,0)$ \\
\hline 12 & 117,3 & - & 7,$06 ; 7,83$ & - \\
\hline 13 & 141,4 & - & 8,$18 ; 7,93$ & - \\
\hline 14 & 130,7 & - & - & - \\
\hline 15 & 132,5 & - & 8,00 & - \\
\hline 16 & 135,8 & - & 8,75 & - \\
\hline $\mathrm{OMe}$ & 56,1 & $3,98(\mathrm{~s})$ & - & \\
\hline
\end{tabular}

\footnotetext{
*Espectro analisado em solução de $\mathrm{CDCl}_{3}$ e constantes de acoplamento J em Hz.
} 
de alcalóides do tipo cantinona também é comum em espécies da família em estudo ${ }^{18}$. Os triterpenos niloticina (3), diidroniloticina (4), taraxerona (5) e o alcalóide 9-metoxi-cantin-6-ona (2) são descritos pela primeira vez na espécie Simaba polyphylla.

\section{AGRADECIMENTOS}

Ao $\mathrm{CNPq}$, pela concessão de bolsas e auxílio à pesquisa pelo programa PNOPG/CNPq (Proc. N. 55026/01-3).

\section{REFERÊNCIAS}

1. Cavalcante, P. B.; Revisão taxonômica do gênero Simaba Aubl. (Simaroubaceae) na América do Sul, Museu Paraense Emílio Goeldi: Belém, 1983, p. 85.

2. Saraiva, R. C. G.; Dissertação de Mestrado, Universidade Federal do Amazonas, Brasil, 2001.

3. Vieira, I. J. C.; Braz Filho, R.; Rodrigues Filho, E.; Vieira, P. C.; Silva, M. F. G. F.; Fernandes, J. B.; J. Braz. Chem. Soc. 1999, 10, 76

4. Ozeki, A.; Hitotsuyanagi, Y.; Hashimoto, E.; Itokawa, H.; Takeya, K.; Alves, S. M.; J. Nat. Prod. 1998, 61, 776.

5. Muhammad, H.; Bedir, E.; Khan, S. I.; Tekwani, B. L.; Khan, I. A.; Takamatsu, S.; Pelletier, J.; Walker, L. A.; J. Nat. Prod. 2004, 67, 772.

6. Arisawa, M.; Fujita, A.; Morita, N.; Kinghorn, A. D.; Cordell; G. A.; Farnsworth, N. R.; Planta Med. 1985, 4, 348.
7. Giesbrecht, A. M.; Gottlieb, H. E.; Gottlieb, O. R.; Goulart, M. O. F.; de Lima, R. A.; Sant'ana, A. E. G.; Phytochemistry 1980, 19, 313.

8. Pohlit, A. M.; Quignard, E. L. J.; Nunomura, S. M.; Tadei, W. P.; Hidalgo; A. F.; Pinto A. C. S.; Santos, E. V. M.; Morais, S. K. R.; Saraiva, R. C. G.; Ming, L. C.; Alecrim, A. M.; Ferraz, A. B.; Pedroso, A. C. S.; Diniz, E. V.; Finney, E. K.; Gomes, E. O.; Dias, H. B.; Souza, K. S.; Oliveira, L. C. P.; Don, L. C.; Queiroz M. M. A.; Henrique, M. C.; Santos, M.; Lacerda Jr, O.; Pinto, P. S.; Silva, S. G.; Graça, Y. R.; Acta Amazonica 2004, 34, 97.

9. Mesquita-Saad, L S. B.; Cabral, J. A.; Acta Amazonica 1997, 27, 269.

10. Gray, A. I.; Bhandari, P.; Waterman, P. G.; Phytochemistry 1988, 27, 1808.

11. Souza, A. F.; Rocha, A. F.; Pinheiro, M. L. B.; Andrade, C. H. S.; Galotta, A. L. A; Santos, M. P. S.; Quim. Nova 2001, 24, 439.

12. Tinto, W. F.; Jagessar, P. K.; Ketwaru, P.; Reynolds, W. F.; McLean, S.; J. Nat. Prod. 1991, 54, 972.

13. Sakurai, N.; Yaguchi, Y.; Inoue, T.; Phytochemistry 1986, 26, 217.

14. Kuo, P.; Shi, L.; Amooru, G. D.; Su, C.; Huang, C., Q., C.; Wu, J.; Lin, A.; Bastow, K. F.; Lee, K.; Wu, T.; J. Nat. Prod. 2003, 66, 1324.

15. McChesney, J. D.; Dou, J. H.; Sindelar, R. D.; Goins, D. K.; Walker, L. A; Rogers, R. D.; J. Chem. Chrystallogr. 1997, 27, 283.

16. Itokawa, H.; Kishi, E.; Morita H; Takeya, K.; Chem. Pharm. Bull. 1992, 40, 1053.

17. Dewick, P. M.; Medicinal Natural Products (A Biosynthetic Approach), John Wiley \& Sons Ltd Ed.: Baffins Lane, Chichester, West Sussex, England, 1997, p. 195, 199, 207.

18. Ohmoto, T.; Koike, K.; The Alkaloids, Arnold Brom Ed.: New York, 1989, p. 135. 